use when the total variances of $M Z$ and DZ twins are unequal. DZ twins had greater total variance for triglycerides. When genetic variance was estimated by subtracting the within-MZ-sets mean square from the within-DZ-sets mean square, all of the lipids had significant estimates of genetic variance; however, when genetic variance was estimated by a method designed to correct bias due to unequal total variances of $\mathrm{MZ}$ and $\mathrm{DZ}$ twins, only triglycerides had significant genetic variance. The heritability of plasma triglycerides was calculated to be 0.6 .

Differential within-twin-pair environmental effects on $\mathrm{MZ}$ and $\mathrm{DZ}$ twins have been postulated to cause differences in total variance. If this is true, previous reports of twins showing significant estimates of genetic variance for plasma cholesterol may be due to environmental bias.

Joe C. Christian, M.D., Department of Medical Genetics, School of Medicine, University of Indiana, Indianapolis, Indiana, USA

\section{A TWIN STUDY ON THE HERITABILITY OF LIPOPROTEIN FRAGTIONS} D. HEWITT, J. MILNER, C. BRECKENRIDGE,
A. J. LITTLE

Department of Epidemiology and Biometrics, University of Toronto, Toronto, Canada

The first investigation to be undertaken by the newly-established Toronto Twin Register is concerned with the heritability of serum lipid concentrations and of specific lipoprotein fractions. Volunteer adult subjects (ages range from 18 to 65), after a 12-hour fast, provide two $15-\mathrm{ml}$ samples of blood for analysis by Auto Analyser II, by agarose electrophoresis and by direct gas chromatography. Provisional diagnosis of zygosity is based on subjects' own opinions, supported by dermatoglyphic analysis and, in any difficult case, substantiated by an enzymatic series.

Analysis of the AA-II and electrophoresis data on the first $2 \times 42$ subjects shows a significant positive correlation within $\mathrm{MZ}$ pairs for each of the Io lipid measurements considered, while the estimate of correlation within DZ pairs is lower in every case. Heritability appears to be higher for cholesterol than for triglyceride fractions. The electrophoretic band for the sinking pre-beta lipoprotein fraction has behaved like a simple genetic marker.

Another aspect of the analysis is the study of lipid differences between $M Z$ cotwins in relation to corresponding intrapair differences in body weight and medical history: male pairs, but not female pairs, show a measurable statistical dependence of cholesterol and triglyceride levels on " excess" body weight.

Dr. Jean Milner, Department of Epidemiology and Biometrics, Toronto 5, Canada

\section{PLASMA PROTEIN VARIABILITY IN MZ TWINS}

\section{MILANI-COMPARETTI, F. SAGCUCCI}

Department of Biology and Genetics, Medical School, University of Ancona, Italy

The Gregor Mendel Institute of Medical Genetics and Twin Research, Rome, Italy

Protein synthesis is under direct genetic control, with environmental influences limited to regulation and to some "high variability" fractions (viz., immunoglobulins). Thus the superimposable genome of MZ twins obviously leads to superimposable protein composition. This can certainly be utilized for zygosity diagnosis.

A previous study had shown that only $\mathrm{MZ}$ twins are easily exchanged by trained police dogs. This is certainly due to the superimposability of the biochemical basis of body scent.

A different approach has now been tried, based on plasma protein fractionating procedures.

A pilot study (in the course of publication elsewhere) has shown that disc Poly Acrilamide Gel Electrophoresis (PAGE) results, as expected, in widely different patterns only in DZ twin pairs.

Plasma specimens from a sample of $60 \mathrm{MZ}$ twin pairs were analysed by PAGE. The substantial superimposability of $\mathrm{MZ}$ cotwin pattern was confirmed. An analysis of the different fractions indicates the extent of environmental variability.

This and other similar methods seem to deserve application in twin zygosity diagnosis and in clinical genetics in general.

Prof. M. Milani-Comparetti, Istituto di Biologia e Genetica dell'Università di Ancona, Italy 